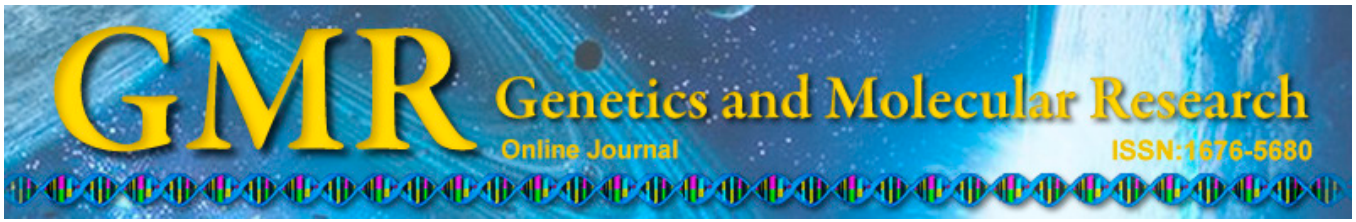

\title{
Investigation of the value of $\mathrm{miR}-21$ in the diagnosis of early stage $\mathrm{HCC}$ and its prognosis: a meta-analysis
}

\author{
S.R. Yan ${ }^{1}$, Z.J. Liu' ${ }^{2}$, S. Yu' ${ }^{1}$ and Y.X. Bao ${ }^{1}$ \\ ${ }^{1}$ Clinical Laboratory Department, \\ The Second Affiliated Hospital of Chongqing Medical University, \\ Chongqing, China \\ ${ }^{2}$ Department of Clinical Medicine, Xinjiang Medical University, \\ Xinjiang, China \\ Corresponding author: Y.X. Bao \\ E-mail: yixibao@163.com
}

Genet. Mol. Res. 14 (3): 11573-11586 (2015)

Received January 30, 2015

Accepted May 29, 2015

Published September 28, 2015

DOI http://dx.doi.org/10.4238/2015.September.28.9

\begin{abstract}
The diagnostic and prognostic value of miR-21 has been examined for hepatocellular carcinoma (HCC), with inconsistent results. Present meta-analysis summarized the diagnostic accuracy and the predictive role for survival of miR-21 in patients with HCC. All eligible studies were searched using PubMed, EMBASE, and Chinese National Knowledge Infrastructure (CNKI) databases up to October 2014. For the diagnostic meta-analysis, the indices of miR-21 in the diagnosis of $\mathrm{HCC}$ were pooled using bivariate random-effect approach models. For the prognostic meta-analysis, data were synthesized with a random effect model, and the hazard ratio (HR) or odd ratio (OR) with its $95 \%$ confidence interval $(95 \% \mathrm{CI})$ was used as the effect size estimate. Ten studies dealing with HCC were included. The overall pooled results for sensitivity, specificity, and the area under the curve (AUC) for the diagnostic meta-analysis (five studies) were $74.0(95 \% \mathrm{CI}=61.0-85.0)$, $78.0(95 \% \mathrm{CI}=67.0-86.0)$, and $0.83(95 \% \mathrm{CI}=0.80-0.86)$, respectively.
\end{abstract}


The combined data for the prognostic meta-analysis (seven studies) suggested that miR-21 overexpression in HCC correlated with poor overall survival $[\mathrm{HR}=1.19(95 \% \mathrm{CI}=0.44-1.94)]$, and higher miR-21 expression was associated with tumor, node, metastases (TNM) stage $[\mathrm{OR}=0.34(95 \% \mathrm{CI}=0.13-0.91)]$. We concluded that miR-21 might be complementary to alpha fetal protein in HCC diagnosis, and might serve as an attractive estimator of HCC. We also demonstrated that miR-21 overexpression was associated with HCC TNM stage and with poor survival. As our study was limited, additional prospective studies are needed to validate these results.

Key words: MiR-21; HCC; Diagnosis; Prognosis; Biomarker

\section{INTRODUCTION}

Hepatocellular carcinoma (HCC) is the third most frequent cause of malignant tumorrelated death worldwide and the second most frequent cause in China (Li et al., 2014). HCC seriously affects the quality of life and health among populations in the world, with the key reason being the lack of accurate molecular markers of diagnosis for the early stages of this disease. Furthermore, due to invasion and intrahepatic metastasis, the prognosis for patients with HCC is poor (Zhou et al., 2013), and the long-term overall survival (OS) rates after clinical treatments remain unsatisfactory because of the high incidence of recurrence and metastasis (Li et al., 2014). The five-year survival rate after surgery for HCC has been reported to be $25-39 \%$ (Li et al., 2014). From the clinical perspective, the molecular factors associated with tumor progression and invasion in HCC have not been well characterized; therefore, it is of marked importance to identify the most useful biomarkers of tumor spreading and of OS as these markers can help guide clinical decision-making with regard to HCC treatment and outcomes (Zhang et al., 2012).

MicroRNAs (miRNAs) comprise a group of small noncoding RNAs which regulate gene expression at the posttranslational level, thereby participating in fundamental biological processes including cell proliferation, differentiation, and apoptosis (Karakatsanis et al., 2013; Wang et al., 2014a; Xie et al., 2014). miRNAs have been proposed to represent ideal biomarker candidates because of their resistance to endogenous RNase resistance and high stability under different storage conditions (Schwarzenbach et al., 2014) and in the peripheral blood (Zhu et al., 2012; Wang et al., 2014c). Abnormal miRNA expression is closely associated with HCC (Tomimaru et al., 2012). Therefore, together with the benefits of their size, abundance, tissue specificity, and relative stability in circulation, miRNAs hold promise as unique accessible biomarkers with potentially important application value for early diagnosis, therapeutic effect monitoring, and the clinical assessment of prognosis in HCC (Ge et al., 2014). mRNA-21 (miR21) has been shown to be significantly upregulated in the circulation of patients with HCC and also in HCC tissues compared with adjacent non-tumor tissues; in addition, miR-21 upregulation was further associated with the capacity of tumor migration and invasion in HCC (Zhu et al., 2012). An increasing number of studies have uncovered a correlation between the expression level of miR-21 and the diagnosis and prognosis of HCC; however, these studies have yielded inconsistent results regarding the diagnostic accuracy and the overall risk for prognosis associated 
with miR-21. To address these issues, we performed this meta-analysis to evaluate the diagnostic efficacy and the prognostic significance of miR-21 in patients with HCC.

\section{MATERIAL AND METHODS}

\section{Search strategy and study selection}

We carefully searched the PubMed, EMBASE, and CNKI databases up to October 2014 to retrieve suitable studies. The keywords for the literature search were as follows: "microRNA-21" or "miR-21" and "HCC" or "hepatocellular carcinoma" and "miRNAs and HCC diagnose or prognosis". Both free text and MeSH searches for keywords were employed. No language limitations were used. Additional studies were obtained by a manual search of the references of relevant reviews. Studies were considered eligible if they met the following criteria: 1) studied patients with HCC; 2) measured expression of miR-21 in tissue or serum or plasma; and 3) investigated the HCC diagnosis or survival or the clinical variables. Articles were excluded based on the following criteria: 1) review articles or systematic reviews, 2) duplicate reports; 3) studies conducted on animals, and 4) conference abstracts or letters to the editors.

\section{Quality assessment}

Two reviewers (S.R.Y. and Y.X.B.) independently assessed the qualities of eligible studies for the diagnostic meta-analysis using the revised quality evaluation tool, Quality Assessment of Diagnostic Accuracy Studies-2 (QUADAS-2) (Whiting et al., 2011), for assessment of the methodological quality of each study. The newly revised tool has been reported to perform better because it offers additional and improved features including distinguishing between bias and applicability, identifying four key domains supported by signaling questions to aid judgment on risk of bias, and rating the risk of bias and concerns about applicability as "high" and "low" (Whiting et al., 2011) regarding the four key domains: patient selection, index test, reference standard, and flow and timing. For the studies included in the prognostic meta-analysis, each was assessed based on the Newcastle-Ottawa scale (NOS) (Wells et al., 2011) for quality assessment. Briefly, the overall star system assessed the three main categories of 1) selection of the cohort, 2) comparability of the cohort, and 3) ascertainment of the outcome. A study could be awarded a maximum of one star for each numbered item within the selection and outcome categories, and a maximum of two stars for comparability. The total number of stars was tallied, with a greater number of awarded stars reflecting a higher methodological quality. A study could be awarded a maximum of nine stars. A flow diagram of the study selection process is presented in Figure 1.

\section{Data extraction and conversion}

Two independent researchers (S.R.Y. and Y.X.B.) performed the data extraction and conversion to reduce inaccuracy in the extracted relevant data from studies included. The title and abstract of each article was reviewed to identify the eligible studies. Disagreements on study eligibility were resolved by full text review and discussion and consensus. The extracted data elements for the diagnostic studies included the following: first author's last 
name, publication year, country, number of patients in experimental and control groups, control type, sample type, test method, area under the curve (AUC), and cutoff values. We extracted the number of patients with true-positive (tp), false-positive (fp), false-negative (fn), and true-negative (tn) test results directly or through calculation based on their corresponding sensitivity and specificity values. We calculated the sensitivity and specificity for each study and analyzed these as bivariate data by methods (Reitsma et al., 2005) for diagnostic metaanalysis. For studies that only provided receiver operating characteristic (ROC) curves without sensitivity and specificity values, the point on the ROC curve which was closest to the left upper corner of the ROC curve space was regarded as the optimal threshold. The data retrieved from prognostic studies included first author last name, publication year, country, number of patients, sample type, tumor, lymph node, metastasis (TNM) stage, test method, cut-off, hazard ratio (HR), and survival. The method consisted of the direct collection of HRs and their $95 \%$ confidence interval $(95 \% \mathrm{CI})$ from the original article. If a direct report of $\mathrm{HR}$ and $95 \% \mathrm{CI}$ was not available, an estimated value was derived indirectly from Kaplan-Meier curves using the methods described by Tierney et al. (2007).

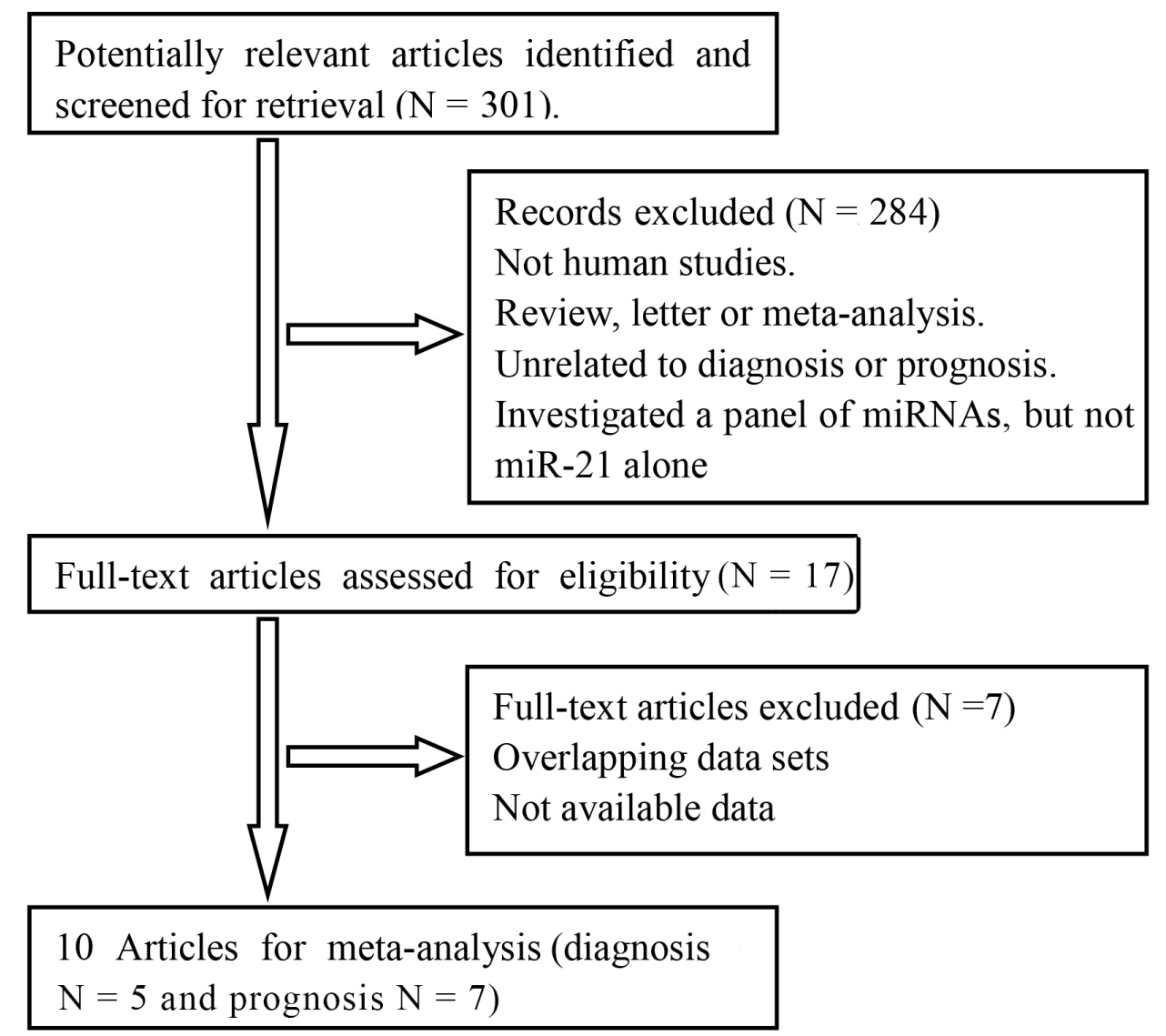

Figure 1. Flow diagram of the study selection process. 


\section{Statistical methods}

For the diagnostic meta-analysis, we calculated the overall pooled sensitivity, specificity, and the summary receiver operating characteristic (sROC) AUCs and their corresponding $95 \%$ CIs by bivariate random-effects approach models. sROC curves (Moses et al., 1993; Walter, 2002) were used to depict the overall diagnostic performance of HCC, as well as the heterogeneity of the eligible studies. As a potential cause of heterogeneity, the threshold/cut off effect was tested using the Spearman correlation coefficient (Devillé et al., 2002). Heterogeneity induced by factors other than the threshold/cut-off effect was assessed by means of the Cochran Q method and also by the test of inconsistency $\left(\mathrm{I}^{2}\right)$ (Higgins and Thompson, 2002). The null hypothesis that the studies were homogeneous was rejected if the $\mathrm{P}$ value for heterogeneity was less than 0.1 or $\mathrm{I}^{2}$ was more than $50 \%$. The Deeks funnel plot asymmetry test was used to evaluate the potential publication bias; $\mathrm{P}>0.05$ for the Deeks test was considered to be representative of a significant statistical publication bias.

For the prognostic meta-analysis, HRs and 95\%CIs were used to estimate the effect of expression on survival, but as these statistical variables were not directly stated in some studies, we estimated their values on the basis of available numerical data derived indirectly from Kaplan-Meier curves using the methods described by Tierney (Tierney et al., 2007) with an HR $>1$ being associated with a poorer outcome. Forrest plots were used to estimate the effect of miR-21 expression on survival outcome. We also investigated the correlation between miR-21 expression and TNM stage. According to the clinical characteristics, stages I and II were combined and stages III and IV were combined (Wang et al., 2014d), with the odd ratio (OR) used as the measure. A test of heterogeneity of the combined HRs was carried out using the Cochran Q test and the Higgins $\mathrm{I}^{2}$ statistic. P values less than 0.1 were considered to be statistically significant. A random effect model was applied if heterogeneity was observed $(\mathrm{P}<0.1)$, while the fixed effect model was used in the absence of betweenstudy heterogeneity $(\mathrm{P}>0.1)$. Publication bias was evaluated using the funnel plot.

All of the above calculations were performed using Stata 12.0 (Stata Corporation, College Station, TX, USA).

\section{RESULTS}

\section{Literature search}

A total of 301 relevant articles were searched from the PubMed, EMBASE, and CNKI databases. After manually screening the titles, abstracts, and keywords, we analyzed the remaining 17 articles by reviewing each full text. Seven articles were omitted because of lack of available data or overlapping data sets. Finally, 10 studies were included, with Tomimaru et al. (2010) and Yu (2012) each counted as two studies because they analyzed the diagnosis as well as the prognosis for miR-21. Of these, five studies were enrolled into the diagnostic meta-analysis (Mizuguchi et al., 2011; Xu et al., 2011; Tomimaru et al., 2012; Yu, 2012; Chen et al., 2014) and seven studies (Tomimaru et al., 2010; Tomimaru et al., 2012; Yu, 2012; Gyöngyösi et al., 2014; Liu et al., 2014; Wang et al., 2014a,b) were included in the prognostic analysis. 


\section{Diagnostic meta-analysis}

\section{Study characteristics}

The diagnostic meta-analysis included five studies published from 2011 to 2014; among these, three were conducted in China and two in Japan. All study test method were quantitative real-time polymerase chain reaction (qRT-PCR). The sample types included serum $(\mathrm{N}=2)$, plasma $(\mathrm{N}=2)$, and tissue $(\mathrm{N}=1)$. There were 985 samples in total, and the numbers of patients with HCC, chronic hepatitis, and cirrhosis, and of healthy controls, were 527, 105, 60, and 293, respectively. Tomimaru et al. (2010) article included two comparisons of sensitivity and specificity (HCC and chronic hepatitis, HCC and healthy controls). The basic characteristics of the studies are outlined in Table 1, and cut-off value, study size, sample, tp, $\mathrm{fp}, \mathrm{tn}$, and fn values for HCC of the five articles are listed in Table 2.

\begin{tabular}{|c|c|c|c|c|c|c|c|}
\hline Study & Location & $\mathrm{HCC}$ & Control type & Controls (N) & AUC & Sensitivity & Specificity \\
\hline Chen (2014) & China & 101 & Healthy & 89 & - & 84.0 & 73.5 \\
\hline $\mathrm{Xu}(2011)$ & China & 42 & Healthy & 42 & $0.910 \pm 0.041$ & 83.1 & 88.1 \\
\hline \multirow[t]{2}{*}{ Tomimaru (2012) } & Japan & 136 & Chronic hepatitis & 30 & $0.773(0.690-0.856)$ & 61.1 & 83.3 \\
\hline & & & Healthy & 50 & $0.953(0.924-0.983)$ & 87.3 & 92.0 \\
\hline Yu (2012) & China & 204 & Chronic hepatitis/cirrhosis/healthy & $75 / 60 / 68$ & - & 45.0 & 58.0 \\
\hline Mizuguchi (2011) & Japan & 44 & Healthy & 44 & $0.78(0.62-0.93)$ & 78.1 & 72.1 \\
\hline
\end{tabular}

$\mathrm{HCC}=$ hepatocellular carcinoma; $\mathrm{AUC}=$ area under the curve.

Table 2. Test method, cut-off value, study size, tp, fp, fn, and tn of miR-21.

\begin{tabular}{|c|c|c|c|c|c|c|c|c|}
\hline Study & Test method & Cut-off & Study size & Sample & tp & $\mathrm{fp}$ & fn & tn \\
\hline Chen (2014) & qRT-PCR & 4.95-fold & 84 & Serum & 34 & 5 & 8 & 37 \\
\hline $\mathrm{Xu}(2011)$ & qRT-PCR & 0.46 & 190 & Serum & 85 & 24 & 16 & 65 \\
\hline \multirow[t]{2}{*}{ Tomimaru (2012) } & qRT-PCR & 0.754 & 216 & Plasm & 77 & 5 & 49 & 25 \\
\hline & & -0.10 & & & 110 & 4 & 16 & 46 \\
\hline Yu (2012) & qRT-PCR & - & 407 & Plasm & 92 & 85 & 112 & 118 \\
\hline Mizuguchi (2011) & qRT-PCR & 10 & 88 & Tissue & 34 & 12 & 10 & 32 \\
\hline
\end{tabular}

tp = true-positive; $\mathrm{fp}=$ false-positive; $\mathrm{fn}=$ true-negative; $\mathrm{tn}=$ false-negative; qRT-PCR = quantitative reverse transcription polymerase chain reaction.

\section{Quality assessments}

We assessed the quality of the five studies according to QUADAS-2. However, none of the studies fulfilled the criteria of the article, as the major bias of the studies was a focus on "patient selection" and "index test". In the domain of patient selection, three studies did not avoid the case-control design, and one study did not state whether a consecutive or random sample of patients was enrolled. In the domain of index testing, none of the studies used a blind method, and in five studies, the index test results were interpreted with knowledge of the results of the reference standard. Two studies pre-specified the threshold, and one study did not describe the threshold specification. Therefore, the overall quality was not satisfactory (Table 3). 
Table 3. Risk of bias and applicability concerns summary.

\begin{tabular}{|c|c|c|c|c|c|c|c|}
\hline \multirow[t]{2}{*}{ Study } & \multicolumn{4}{|c|}{ Risk of bias } & \multicolumn{3}{|c|}{ Applicability concerns } \\
\hline & Patient selection & Index test & Rreference standard & Flow and timing & Patient selection & Index test & Reference standard \\
\hline Chen (2014) & - & $?$ & + & + & + & + & + \\
\hline $\mathrm{Xu}(2011)$ & - & - & + & $?$ & + & + & + \\
\hline Tomimaru (2012) & $?$ & - & + & + & + & + & + \\
\hline $\mathrm{Yu}(2012)$ & + & $?$ & + & + & + & $?$ & + \\
\hline Mizuguchi (2011) & + & $?$ & + & + & + & + & + \\
\hline
\end{tabular}

$(-)=$ high risk; $(?)=$ unclear; $(+)=$ low risk.

\section{Summary of the diagnostic accuracy of miR-21 for HCC}

The sensitivity and the specificity observed in the five diagnostic studies ranged from 87.3-45.0\% and $92.0-58.0 \%$, respectively. After analysis of the bivariate random effect model, the pooled sensitivities and specificities were $74.0 \%(95 \% \mathrm{CI}=61.0-85.0)$ and $78.0 \%(95 \% \mathrm{CI}$ $=67.0-86.0$ ), respectively. For the bivariate sROC curve for the diagnostic accuracy of miR21 , the AUC was $0.83(95 \% \mathrm{CI}=0.80-0.86)$. The sROC curve also showed the summary point estimate of sensitivity and specificity. The wider region ( $95 \%$ confidence contour) demonstrated more uncertainty as to where the likely values of sensitivity and specificity might occur for individual studies; the smaller region (95\% confidence contour) contained likely combinations of the mean values of sensitivity and specificity. The sROC curve for miR-21 is shown in Figure 2.

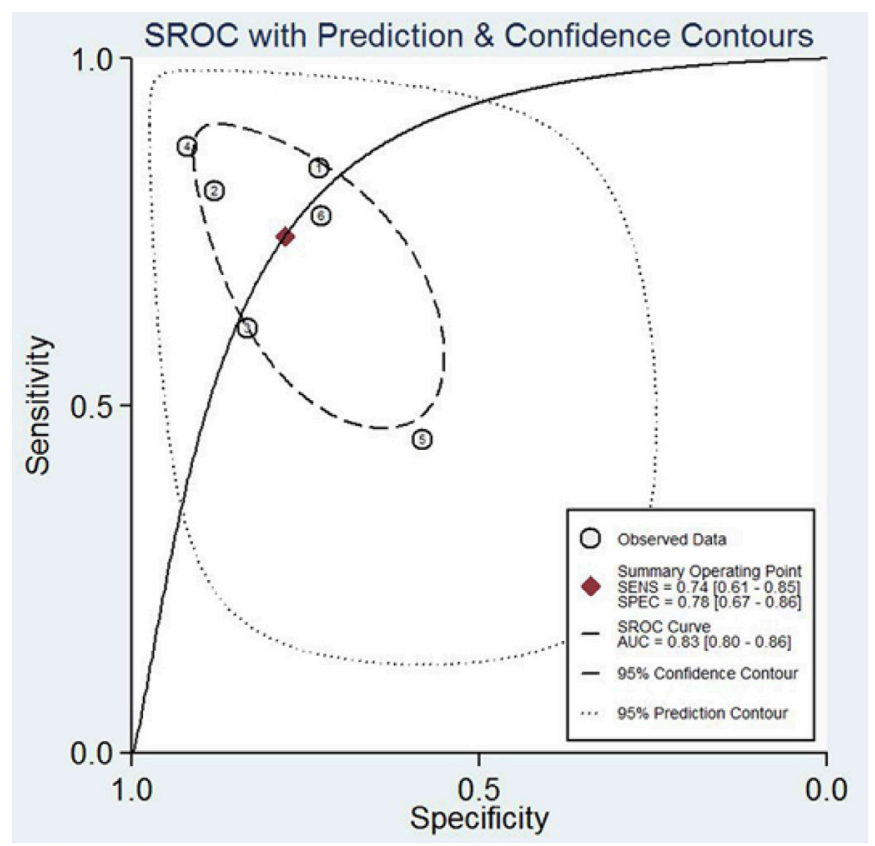

Figure 2. sROC curves from the bivariate model. The smaller region (confidence contour) contains likely combinations of the mean values of sensitivity and specificity. The wider region (prediction contour) demonstrates more uncertainty as to where the likely values of sensitivity and specificity might occur for individual studies. $\mathrm{sROC}=$ summary receiver operating characteristic; SENS = sensitivity; SPEC = specificity. 


\section{Test of heterogeneity for miR-21}

The plane scatter distribution did not present a typical "shoulder shape", and the Spearman correlation coefficient was -0.600 ; the $\mathrm{P}$ value was 0.208 . These indicated that threshold effects did not exist in our analysis. By means of the Cochran Q method and the $\mathrm{I}^{2}$ test, the values of Cochran- $\mathrm{Q}=2.525, \mathrm{P}=0.141 ; \mathrm{I}^{2}=20.79(95 \% \mathrm{CI}=0.00-100)$ demonstrated the lack of heterogeneity in the data. The Deeks test indicated that there was none publication bias in the meta-analysis $(\mathrm{P}=0.094)$ (Figure 3$)$.

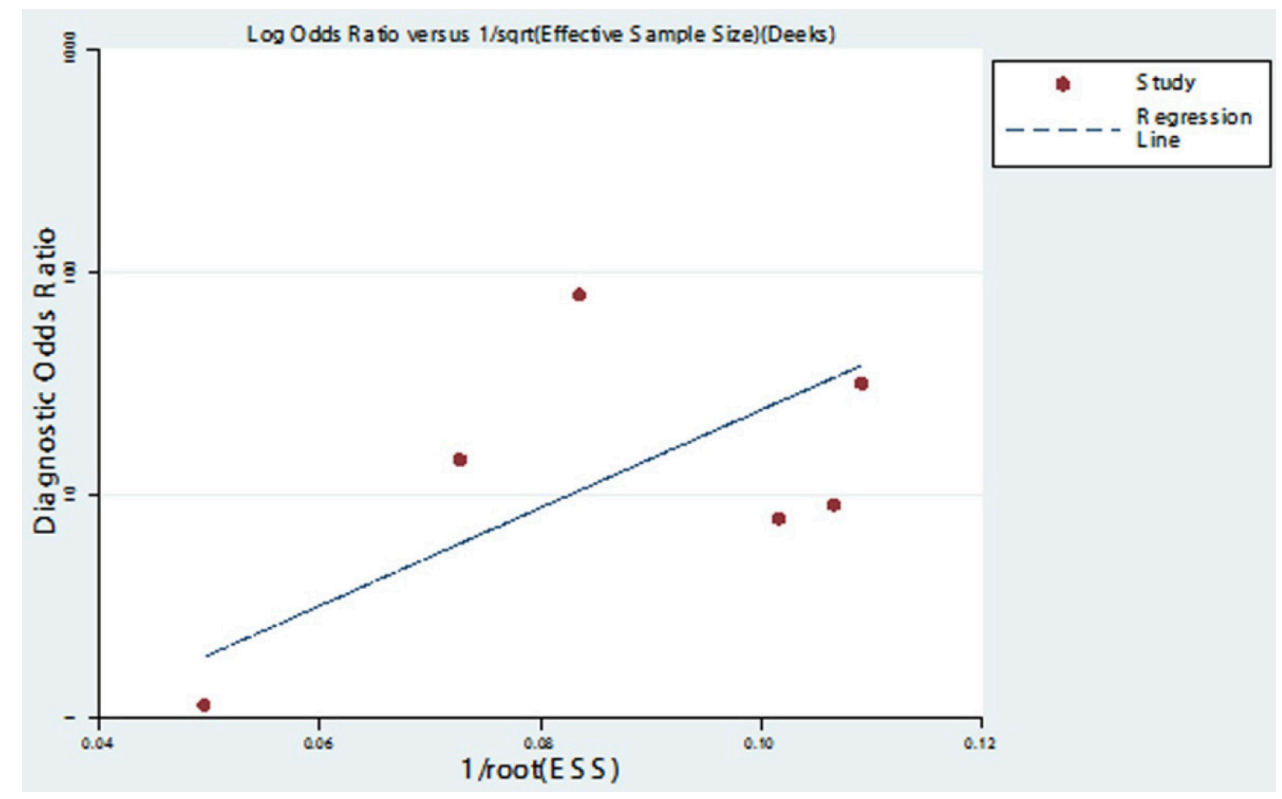

Figure 3. Deeks' funnel plot based on the data of the five studies. ESS, effective sample size.

\section{Prognostic meta-analysis}

\section{Study characteristics}

The seven studies eligible for prognostic analysis were published from 2010 to 2014 and included a total of 723 patients; four studies were performed in China (Yu, 2012; Liu et al., 2014; Wang et al., 2014a,b), one in Hungary (Gyöngyösi et al., 2012), one in Japan (Tomimaru et al., 2012), and one in the United Kingdom (Tomimaru et al., 2010). The types of specimen included tissues $(\mathrm{N}=4)$ and serum $(\mathrm{N}=3)$. In seven studies, the universal method used for detecting miR-21 expression was qRT-PCR for all studies. Four studies (Gyöngyösi et al., 2012; Yu, 2012; Liu et al., 2014; Wang et al., 2014a) provided data on OS and four studies (Tomimaru et al., 2010, 2012; Wang et al., 2014a,b) went on to demonstrate the connection between miR-21 expression and HCC TNM staging; however, the cut-off values of miR-21 used appeared to be different. The main features of the eligible studies are summarized in Table 4. 
Table 4. Main characteristics of seven studies in the prognosis meta analysis.

\begin{tabular}{llcclcccc}
\hline Study & Location & NOS & Patients $(\mathrm{N})$ & Sample & TNM stage & Cut-off & HR & Survival \\
\hline Wang (2014a) & China & 8 & 119 & Tissues & I-IV & Median & Reported & OS \\
Gyöngyösi (2014) & Hungary & 5 & 20 & Tissues & I-IV & Median & Reported & OS \\
Yu (2012) & China & 6 & 252 & Tissues & I-IV & Median & Reported & OS \\
Liu (2014) & China & 7 & 136 & Serum & - & Median & Reported & OS \\
Wang (2014b) & China & 6 & 30 & Serum & I-IV & 5-Fold & - & - \\
Tomimaru (2012) & Japan & 6 & 136 & Serum & I-IV & $0.754($ T) & - & - \\
Yomimaru (2010) & UK & 5 & 30 & Tissues & I-IV & Median & - & - \\
\hline
\end{tabular}

$\mathrm{TNM}=$ tumor node metastasis; $\mathrm{HR}=$ hazard ratio; $\mathrm{OS}=$ overall survival.

\section{Quality assessments}

To assess the quality of the eligible studies, two authors independently extracted data and assessed the methodological quality using the NOS. The scores are shown in Table 1. The points of study quality assessed by the NOS ranged from 5 to 8 (with a mean of 6.14), with higher value indicating better methodology. The studies included in our meta-analysis all had high levels of methodological quality ( $>5$ stars on the NOS) (Wells et al., 2011).

\section{Correlation between miR-21 expression and $O S$}

All four studies reported data on miR-21 expression and OS in HCC; two presented information on miR-21 expression and OS in HCC initially treated by sorafenib (Gyöngyösi et al., 2012) and transarterial chemoembolization (Liu et al., 2014). High miR-21 expression was found to be significantly correlated with poor OS, with a pooled HR estimate of 1.19 (95\%CI $=0.44-1.94$ ), (Figure 4). The heterogeneity of the four studies was $\mathrm{P}=0.00, \mathrm{I}^{2}=94.0 \%$; therefore, heterogeneity was observed in the data.

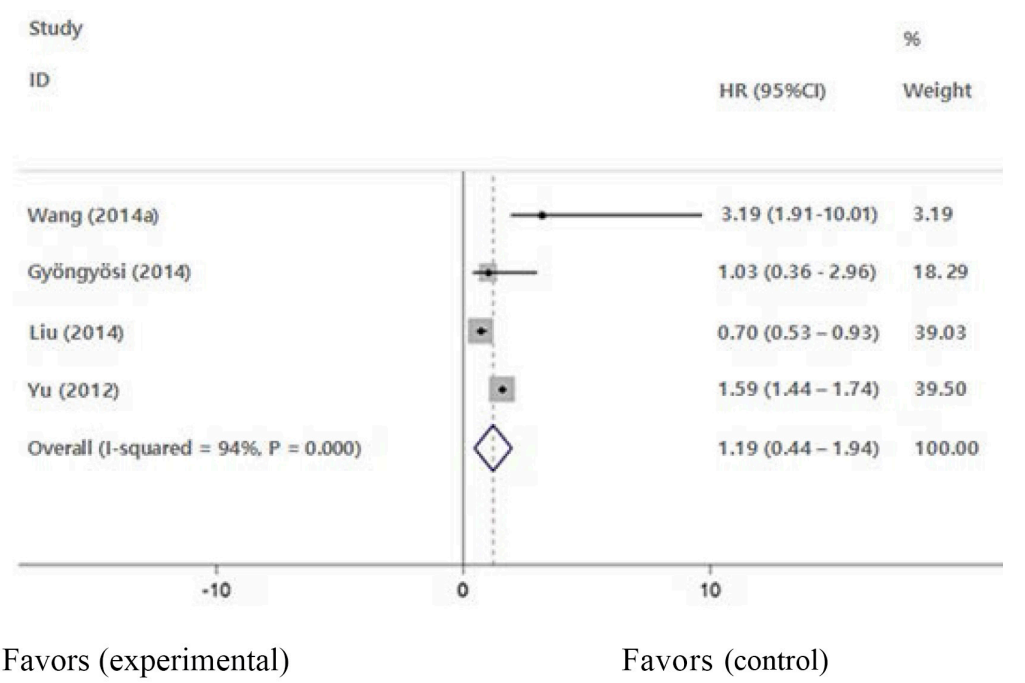

Figure 4. Meta-analysis of the association between miR-21 over-expression and the OS of HCC. Each study is shown by the name of the first author and the HR with $95 \%$ CIs. The summary HR and $95 \%$ CIs are also shown (according to the random effects estimations). $\mathrm{OS}=$ long term overall survival; $\mathrm{HCC}=$ hepatocellular carcinoma; $\mathrm{HR}=$ hazard ratio. 


\section{miR-21 expression and TNM stage in HCC}

Four studies reported data on miR-21 expression and TNM stage in HCC; these were pooled to calculate the ORs. In our study, we found that miR-21 expression was significantly correlated with TNM stage. The ORs and the corresponding CIs of the studies reporting TNM stages were $0.34(95 \% \mathrm{CI}=0.13-0.91)$, (Figure 5); in these, the heterogeneity was evident, $\mathrm{I}^{2}=69.1 \%, \mathrm{P}=0.021$.

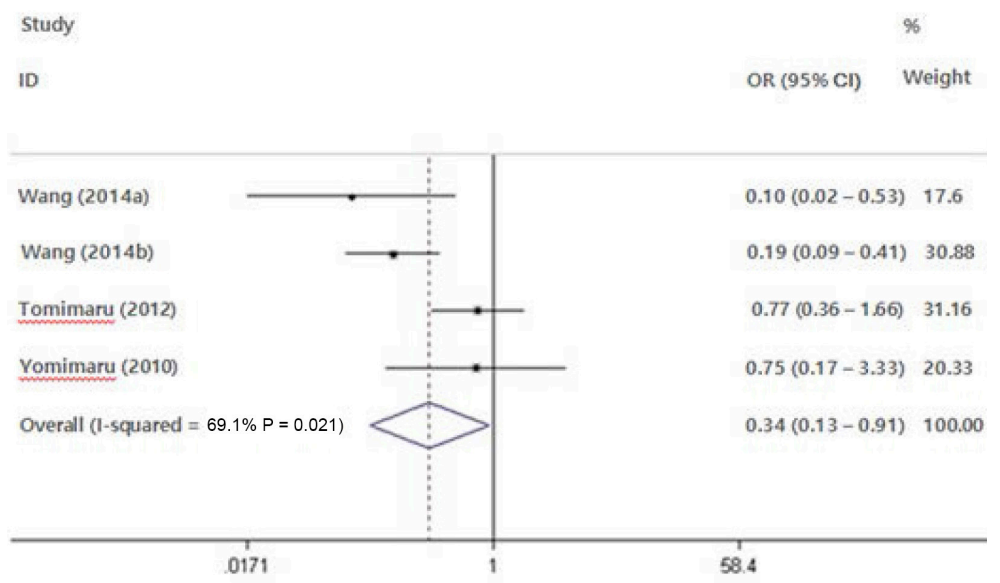

Favors (experimental) Favors (control)

Figure 5. Summary of the correlations of miR-21 expression and TNM (tumor, lymph node, metastasis) stage. Estimated odds ratios (ORs) are shown.

\section{Publication bias}

Finally, the publication bias of the studies included was evaluated by funnel plots and the Egger tests. However, as shown in Figure 6, the funnel plots showed asymmetry, and the P value of the Egger test was 0.696 (greater than 0.1), which suggested that the risk of publication bias did not exist in the meta-analysis.

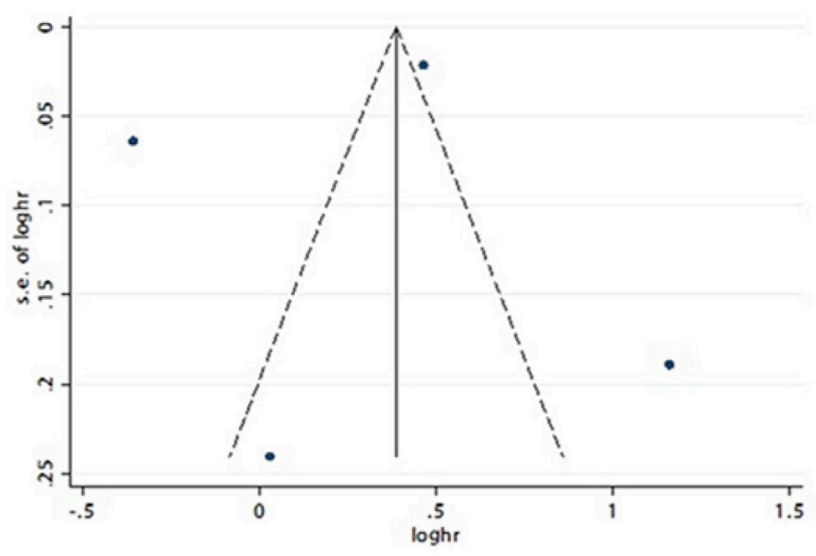

Figure 6. Funnel plot with pseudo $95 \%$ confidence limits. s.e. $=$ standard error. 


\section{DISCUSSION}

Tumor metastasis and recurrence often contribute to the poor clinical outcome of HCC patients. Ultrasonography and serum alpha fetal protein (AFP) are widely used to detect early stage HCC in the clinic, but because of the low sensitivity, the clinical efficacy of AFP for disease diagnosis is compromised. Therefore, the development of suitable biomarkers is critical for cancer diagnosis or the prediction of patient outcome. Recently, circulating miRNAs have attracted considerable attention for their high stability and marked potential as biomarkers. Although the association of miR-21 with $\mathrm{HCC}$ has been explored over the past several years, circulating miRNAs have increasingly come into focus as a potential biomarker for HCC diagnosis, prognosis, and efficacy of treatments. However, the conclusions reached in the literature regarding diagnostic accuracy and prognostic ability have been inconsistent or even contradictory. As the available data have not yet been analyzed comprehensively, we therefore carried out these meta-analyses to study the correlations of miR-21 expression with HCC prognosis and diagnosis.

The present diagnostic meta-analysis of HCC has shown that, as a diagnostic biomarker, circulating miR-21 achieved a pooled sensitivity of $74.0 \%$ and a specificity of $78.0 \%$. Whereas the sensitivity and specificity were not high for circulating miR-21, as compared with currently utilized diagnostic tools, the sensitivity found was higher than those obtained by computed tomography or magnetic resonance imaging. In addition, the AUC of miR-21 was slightly higher than that of AFP ( 0.83 vs 0.8081$)$; together with the overall AUC, the value of the sROC curve can be used as an index to assess the discrimination ability of a given marker (Jones and Athanasiou, 2005). Furthermore, circulating miR-21 also shows many advantages compared with histopathological examination, most notably that its detection does not require an invasive or harmful procedure. Wang et al. (2014c) also demonstrated that serum miR-21 was a significant independent factor for HCC recurrence, and this measure was also reported to be more sensitive than AFP for the detection of HCC. This observation suggests that serum miRNAs, such as miR-21, might be useful for the prediction of HCC risk and the early detection of HCC (Wang et al., 2014a). Multiple reports have suggested that a panel of several miRNAs in combination might significantly improve diagnostic accuracy as compared with that obtained from a single miRNA; such an mRNA panel might be effective in differentiating HCC from healthy, chronic hepatitis B, and cirrhosis individuals with a high degree of accuracy (Yu, 2012; Tan et al., 2014). Studies also have been demonstrated that such an mRNA panel has considerable clinical value for early HCC diagnosis and the diagnosis of AFP-negative HCC (Jones and Athanasiou, 2005; Yu, 2012). The value of miR21 and AFP screening in combination still requires further research. The combined detection of AFP and miR-21, and a combination with clinical symptoms and imaging data might be achieved a better diagnostic accuracy to achieve a definitive diagnosis. We further assessed the role of circulating miR-21 in prognosis. Meta-analysis showed that elevated miR-21 could predict poor survival in patients with HCC, and that patients with an increased level of miR21 had a higher risk of poor survival, which was 1.19 fold higher than the risk in patients with a low miR-21 level. We believe that miR-21 could serve as a potential biomarker for prognosis; however, the reasons underlying the differences of OS among patients with HCC and high or low miR-21 expression remain unclear. Notably, tumor invasion and metastasis have also generally been correlated with the poor prognosis of HCC (Tung-Ping Poon et al., 2000). Our meta-analysis demonstrated that miR-21 overexpression is in turn positively related to invasion-related parameters such as tumor grade, as reflected by a high-grade of 
the TNM stage $(\mathrm{OR}=0.34 ; 95 \% \mathrm{CI}=0.13-0.91)$. However, between-study heterogeneity was observed, and the meta-regression could not be performed because of the small number of primary studies included for analysis. We suggest that variation among the different races and sample types among the studies might be a potential source of the detected heterogeneity. Many studies have supported the hypothesis that miR-21 overexpression might promote HCC invasion and metastasis through direct or indirect mechanisms, and thus lead to poor prognosis (Liu et al., 2010; Tomimaru et al., 2010; Xu et al., 2011; Tomimaru et al., 2012; Zhu et al., 2012). Zhou et al. (2013) demonstrated that miR-21 promoted side population cell migration and invasion by targeting PTEN, RECK, and PDCD4, and that it could also directly target MAP2K3 and inhibit its expression during HCC carcinogenesis (Xu et al., 2013). Therefore, patients with high tumoral miRNA-21 expression were shown to have a significantly shorter time-to-recurrence compared to those with low tumoral expression, and that patients with low plasma miRNA-21 expression tended to have a longer time-to-recurrence compared to those with high plasma miRNA-21 expression (Tomimaru et al., 2012).

Advantages of this systematic review include its well defined search strategy, rigorous selection of study literature according to the inclusion criteria, the enrollment of subjects with chronic hepatitis, cirrhosis, and healthy constitutions as "controls" in the studies used for diagnostic meta-analysis, and the consistency of the method used to detect circulating miRNAs between studies. However, some limitations of this meta-analysis should be addressed. First, in this analysis, the total number of studies included and the total sample size were relatively small; which might influence the validity of our analysis to some extent; second, the prognosis meta-analysis demonstrated concerns regarding heterogeneity, although most of these results were not highly significant. These or other unrecognized limitations might possibly have influenced the results of this meta-analysis; therefore, our results should be interpreted with caution and the conclusions of this meta-analysis should be carefully drawn.

From the results of these meta-analyses, we concluded that miR-21 might serve as a complementary marker to AFP in HCC diagnosis. Although miR-21 did not show the excellent value of the other imaging technologies, miR-21 is still an attractive estimator of HCC. We also demonstrated that miR-21 overexpression was associated with the poor survival of patients with HCC and with their TNM stage. However, as our review inevitably had a number of limitations, additional prospective studies are needed in the future to verify our findings.

\section{Conflicts of interest}

The authors declare no conflict of interest.

\section{ACKNOWLEDGMENTS}

Research supported by the funds of National Natural Science Foundation of China (\#81274144 and \#81473388).

\section{REFERENCES}

Chen Z, Ding WB, Miao YJ, Wang YF, et al. (2014). The changes of serum miR-21 expression level in patients with HCC before and after TACE and its clinical significance. J. Intervent. Radiol. 23: 406-410.

Devillé WL, Buntinx F, Bouter LM, Montori VM, et al. (2002). Conducting systematic reviews of diagnostic studies: didactic guidelines. BMC Med. Res. Methodol. 2: 9. 
Ge W, Yu DC, Li QG, Chen X, et al. (2014). Expression of serum miR-16, let-7f, and miR-21 in patients with hepatocellular carcinoma and their clinical significances. Clin. Lab. 60: 427-434.

Gyöngyösi B, Végh E, Járay B, Székely E, et al. (2014). Pretreatment microRNA level and outcome in sorafenib-treated hepatocellular carcinoma. J. Histochem. Cytochem. 62: 547-555.

Higgins JP and Thompson SG (2002). Quantifying heterogeneity in a meta-analysis. Stat. Med. 21: 1539-1558.

Jones CM and Athanasiou T (2005). Summary receiver operating characteristic curve analysis techniques in the evaluation of diagnostic tests. Ann. Thorac. Surg. 79: 16-20.

Karakatsanis A, Papaconstantinou I, Gazouli M, Lyberopoulou, et al. (2013). Expression of microRNAs, miR-21, miR31, miR-122, miR-145, miR-146a, miR-200c, miR-221, miR-222, and miR-223 in patients with hepatocellular carcinoma or intrahepatic cholangiocarcinoma and its prognostic significance. Mol. Carcinog. 52: 297-303.

Li J, Gao JZ, Du JL and Wei LX (2014). Prognostic and clinicopathological significance of glypican-3 over expression in hepatocellular carcinoma: A meta-analysis. World J. Gastroenterol. 20: 6336-6344.

Liu C, Yu J, Yu S, Lavker RM, et al. (2010). MicroRNA-21 acts as an oncomir through multiple targets in human hepatocellular carcinoma. J. Hepatol. 53: 98-107.

Liu M, Liu J, Wang L, Wu H, et al. (2014). Association of serum microRNA expression in hepatocellular carcinomas treated with transarterial chemoembolization and patient survival. PLoS One 9: e109347.

Mizuguchi Y, Mishima T, Yokomuro S, Arima Y, et al. (2011). Sequencing and bioinformatics-based analyses of the microRNA transcriptome in hepatitis B-related hepatocellular carcinoma. PLoS One 6: e15304.

Moses LE, Shapiro D and Littenberg B (1993). Combining independent studies of a diagnostic test into a summary roc curve: data-analytic approaches and some additional considerations. Stat. Med. 12: 1293-1316.

Reitsma JB, Glas AS, Rutjes AW and Scholten RJ (2005). Bivariate analysis of sensitivity and specificity produces informative summary measures in diagnostic reviews. J. Clin. Epidemiol. 58, 982-990.

Schwarzenbach H, Nishida N, Calin GA and Pantel K (2014). Clinical relevance of circulating cell-free microRNAs in cancer. Nat. Rev. Clin. Oncol. 11: 145-156.

Tan Y, Ge G, Pan T, Wen D, et al. (2014). A serum microRNA panel as potential biomarkers for hepatocellular carcinoma related with hepatitis B virus. PLoS One 9: e107986.

Tierney JF, Stewart LA, Ghersi D, Burdet S, et al. (2007). Practical methods for incorporating summary time-to-event data into meta-analysis. Trials 8: 16.

Tomimaru Y, Eguchi H, Nagano H, Wada H, et al. (2010). MicroRNA-21 induces resistance to the anti-tumour effect of interferon- $\alpha / 5$-fluorouracil in hepatocellular carcinoma cells. Br. J. Cancer 103: 1617-1626.

Tomimaru Y, Eguchi H, Nagano H, Wada H, et al. (2012). Circulating microRNA-21 as a novel biomarker for hepatocellular carcinoma. J. Hepatol. 5: 167-175.

Tung-Ping Poon R, Fan ST and Wong J (2000). Risk factors, prevention, and management of postoperative recurrence after resection of hepatocellular carcinoma. Ann. Surg. 232: 10-24.

Walter SD (2002). Properties of the summary receiver operating characteristic (SROC) curve for diagnostic test data. Stat. Med. 21: 1237-1256.

Wang H, Hou L, Li A, Duan Y, et al. (2014a). Expression of serum exosomal microRNA-21 in human hepatocellular carcinoma. Biomed Res. Int. 2014: 864894.

Wang WY, Zhang HF, Wang L, Ma YP, et al. (2014b). miR-21 expression predicts prognosis in hepatocellular carcinoma. Clin. Res. Hepatol. Gastroenterol. 38: 715-719.

Wang Y, Gao X, Wei F, Zhang X, et al. (2014c). Diagnostic and prognostic value of circulating miR-21 for cancer: A systematic review and meta-analysis. Gene 533: 389-397.

Wang Z, Cia Q, Jiang Z, Liu B, et al. (2014d). Prognostic role of microRNA-21 in gastric cancer: A meta-analysis. Med. Sci. Monit. 20: 1668-1674.

Whiting PF, Rutjes AW, Westwood ME, Mallett S, et al. (2011). QUADAS-2: a revised tool for the quality assessment of diagnostic accuracy studies. Ann. Intern. Med. 155: 529-536.

Wells GA, Shea B, O'Connell D, Peterson J, et al. (2011). The Newcastle-Ottawa Scale (NOS) for assessing the quality of nonrandomized studies in meta-analysis. Available at [www.ohri.ca/programs/clinical_epidemiology/oxford.asp]. Accessed November 25, 2012.

Xie KL, Zhang YG, Liu J, Zeng Y, et al. (2014). MicroRNAs associated with HBV infection and HBV-related HCC. Theranostics 4: 1176-1192.

Xu G, Zhang Y, Wei J, Jia W, et al. (2013). MicroRNA-21 promotes hepatocellular carcinoma HepG2 cell proliferation through repression of mitogen-activated protein kinase-kinase 3. BMC Cancer 13: 469.

Xu J, Wu C, Che X, Wang L, et al. (2011). Circulating microRNAs, miR-21, miR-122, and miR-223, in patients with hepatocellular carcinoma or chronic hepatitis. Mol. Carcinog. 50: 136-142. 
Yu L (2012). Investigated the value of miR-21 in diagnosis early-stage of HCC and the prognosis of HCC. Ph.D. dissertation, University of Fudan.

Zhang CH, Xu GL, Jia WD, Ge YS, et al. (2012). Prognostic significance of osteopontin in hepatocellular carcinoma: a meta-analysis. Int. J. Cancer 130: 2685-2692.

Zhou L, Yang ZX, Song WF, Li QJ, et al. (2013). MicroRNA-21 regulates the migration and invasion of a stem-like population in hepatocellular carcinoma. Int. J. Oncol. 43: 661-669.

Zhu Q, Wang Z, Hu Y, Li J, et al. (2012). miR-21 promotes migration and invasion by the miR-21 -PDCD4-AP-1 feedback loop in human hepatocellular carcinoma. Oncol. Rep. 27: 1660-1668. 